\title{
An engineering model for 3-D turbulent wind inflow based on a limited set of random variables
}

\author{
Manuel Fluck and Curran Crawford \\ Department of Mechanical Engineering, Institute for Integrated Energy \\ Systems (IESVic), University of Victoria, Victoria, BC, Canada \\ Correspondence to: Manuel Fluck (mfluck@uvic.ca)
}

Received: 31 January 2017 - Discussion started: 1 March 2017

Revised: 25 September 2017 - Accepted: 10 October 2017 - Published: 16 November 2017

\begin{abstract}
Emerging stochastic analysis methods are of potentially great benefit for wind turbine power output and loads analysis. Instead of requiring multiple (e.g. $10 \mathrm{~min}$ ) deterministic simulations, a stochastic approach can enable a quick assessment of a turbine's long-term performance (e.g. 20-year fatigue and extreme loads) from a single stochastic simulation. However, even though the wind inflow is often described as a stochastic process, the common spectral formulation requires a large number of random variables to be considered. This is a major issue for stochastic methods, which suffer from the "curse of dimensionality" leading to a steep performance drop with an increasing number of random variables contained in the governing equations. In this paper a novel engineering wind model is developed which reduces the number of random variables by 4-5 orders of magnitude compared to typical models while retaining proper spatial correlation of wind speed sample points across a wind turbine rotor. The new model can then be used as input to direct stochastic simulations models under development. A comparison of the new method to results from the commercial code TurbSim and a custom implementation of the standard spectral model shows that for a 3-D wind field, the most important properties (cross-correlation, covariance, auto- and cross-spectrum) are conserved adequately by the proposed reducedorder method.
\end{abstract}

\section{Introduction}

Engineering design tasks frequently face uncertain or random model parameters (e.g. imprecise component geometries), system properties (e.g. tolerances on manufacturing quality), and/or boundary conditions (e.g. varying wind conditions). In a deterministic modelling framework, the analysis of such uncertain systems produces one specific solution for each realization of the random quantity. A "realization" (also referred to as one "sample") is one specific observation of the random quantity, for example a specific solution for one specific geometry or one specific set of inflow conditions. In a numerical experiment, a realization is usually obtained from one specific random seed. However, through this process the stochastic dimension of the problem at hand is either ignored entirely, by analyzing the most likely case only (the purely deterministic approach), or it requires multiple parallel solutions to asses the statistics of the results a posteriori, for example via extreme value, sensitivity analysis, or Monte Carlo simulation. Often the first two options are insufficient, and the latter is computationally too expensive. To solve this dilemma the focus of recent research has lately moved towards stochastic analyses and uncertainty quantification (Sudret, 2007; Najm, 2009; Le Maître and Knio, 2010; Sullivan, 2015). Rather than generating one specific solution for each realization of a random input or model quantity, a stochastic analysis can help assess uncertainties quicker and even include uncertain quantities directly into the system analysis. A stochastic analysis may not only provide one specific solution but may also solve the problem for the whole ensemble of all possible realizations at once; see Fig. 1. Thus, it becomes possible to transform the problem from multiple deterministic realizations with random seeds to a stochastic formulation of the governing equations that directly handles the stochastic variables in the system. The stochastic solution then directly describes the statistics (e.g. the probability 


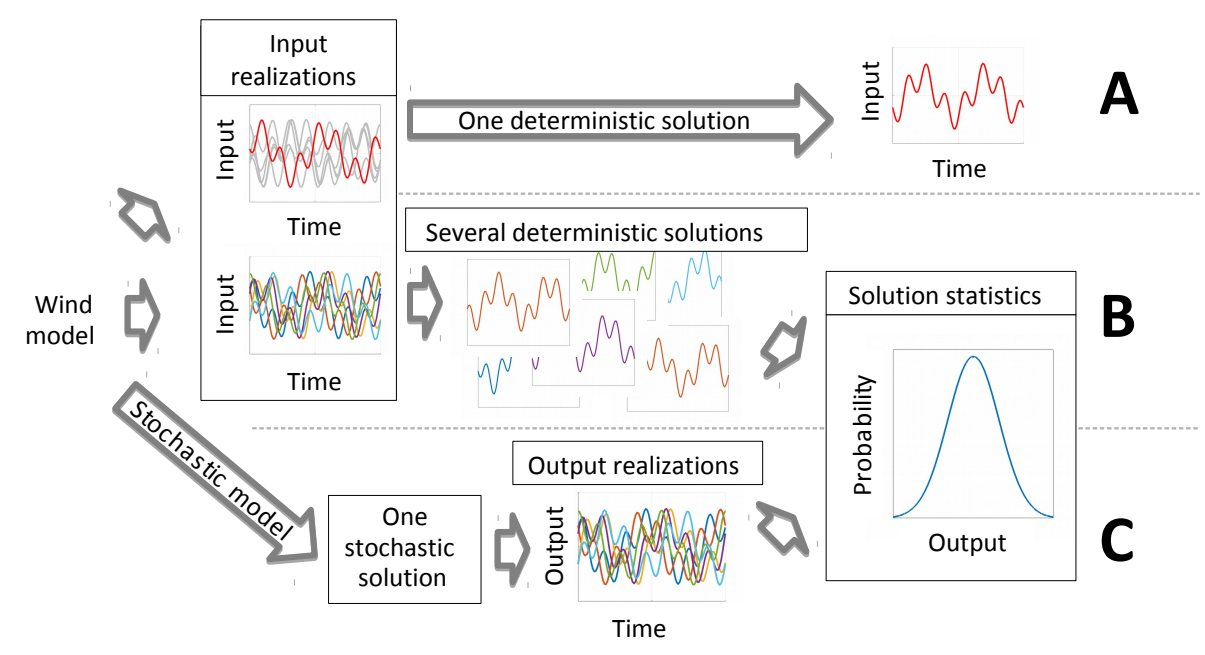

Figure 1. Comparison of the solution processes in a pure deterministic (A), a deterministic-statistic (B), and a stochastic framework (C).

distributions) of the outputs, based on the properties given for the input variables in the forcing terms of the governing system equations.

In wind turbine engineering, the driving force is the turbulent atmospheric wind which is commonly described as a stochastic field derived from turbulent wind models developed around stochastic 10 min mean wind speed distributions. This naturally invites the use of stochastic methods to asses extreme and fatigue loads, annual power production, power fluctuations, etc., in a stochastic sense and thus exploit the advantages of stochastic methods. However, wind turbine design and analysis are usually carried out in a deterministic fashion or at best as a Monte Carlo-like set of several subsequent deterministic solutions (path A and B in Fig. 1, respectively). The wind turbine design standard IEC 614001, Ed. 3 (2005) is indicative of this deterministic framework. It bases the turbine load analysis on multiple deterministic simulations, carried out at many different mean wind speeds, for about 20 different load cases, each simulated for $10 \mathrm{~min}$ and repeated several times with different realizations of the turbulent inflow, each generated from a different numerical random seed. For a land-based turbine this quickly amounts to evaluating several hundred $10 \mathrm{~min}$ samples. For offshore turbines, where various wind conditions (wind speed and direction) additionally have to be combined with various sea states (combinations of wave height and direction), this number increases to several thousand $10 \mathrm{~min}$ evaluations. However, even with a large number of deterministic simulations, extrapolation to extreme loads is a delicate exercise and results can vary greatly (Moriarty, 2008; Burton et al., 2011). For a single load case and one mean wind speed bin, Zwick and Muskulus (2015) show that basing a wind turbine analysis on six $10 \mathrm{~min}$ wind speed simulations, generated from six different random seeds, results in a difference of up to $34 \%$ in the ultimate loads for the most extreme $1 \%$ of seed combinations. Tibaldi et al. (2014) present a study which indicates that turbine loads extracted even from 20 different $10 \mathrm{~min}$ wind fields, generated from 20 different random seeds, vary greatly. This shows that in a deterministic framework, load variations from different random seeds can dominate effects from design parameter changes even with a fairly large number of realizations analyzed. Obviously this constitutes a severe problem, particularly when concerned with gradientbased optimization where not only relatively fast solutions times but also reliable design variable gradients are vital.

A direct stochastic treatment of the wind loading (path $\mathrm{C}$ in Fig. 1), on the other hand, considers the wind as a stochastic process throughout the turbine simulation procedure. It postpones the generation of realizations until after the calculation of a solution for the system equations, which thus become stochastic equations. Hence, it can be a means to efficiently include stochastic parameters, directly obtain a stochastic solution, and arrive at the statistics of the resulting loads much more quickly. Fluck and Crawford (2017a) present an example of a stochastic analysis for wing loads in turbulent inflow, and show that such a stochastic approach does not rely on the repeated analysis of multiple (e.g. $600 \mathrm{~s}$ ) realizations of the wind field. Instead, one (possibly short, e.g. $10 \mathrm{~s}$ ) stochastic result yields all possible realizations and hence contains the full spectrum of uncertainties. Thus, it will enable the analyst to obtain a more complete description of the resulting load ensemble at large, calculate its statistics, and eventually arrive at more precise estimates of, e.g., the probability of the exceedance of some load threshold more quickly.

Recently, progress has been made towards stochastic analysis of wind turbines. For example, results have been shown for an aeroelastic analysis with one uncertain system parameter - stiffness or damping (Desai and Sarkar, 2010) - and for a stochastic formulation of airfoil lift, drag, and pitching moment in stall conditions (Bertagnolio et al., 2010). Moreover, stochastic models have been used for wake modelling, treating wake center and shape as random processes 
(Doubrawa et al., 2017). However, only very early steps have been completed to include the biggest source of uncertainty: the uncertain inflow from turbulent atmospheric wind. On a wind farm scale, Padrón et al. (2016) recently presented a layout optimization based on a polynomial chaos formulation for the freestream wind speed and direction. Guo (2013) offers a stochastic wind model used for a stochastic analysis of wind turbine loads. However, he still bases the stochastic analysis on deterministic sampling (i.e. path B in Fig. 1). Moreover, this model is driven by the decomposition (biorthogonal and Karhunen-Loève) of a specific set of wind field data. Hence, it is not generally applicable but relies on the availability of sufficient data.

As turbulent wind is already represented as a stochastic field in many common wind models, a transition from a deterministic aerodynamic model for specific wind realizations to a stochastic model yielding the whole stochastic load ensemble at once seems an obvious step. However, this step comes with a simple, yet fundamental challenge: current wind models, even simple spectral models, rely on a large number of random variables to set the wind sample's phase angles. Since realizations of large sets of random variables can be generated very quickly, this is not a problem for deterministic load analyses. However, the computational cost of stochastic analysis methods increases dramatically with the number of random variables included, a fact commonly known as the "curse of dimensionality" (Majda and Branicki, 2012). This renders current wind models inaccessible to stochastic methods and thus poses a major barrier to the further development of stochastic models for the analysis of wind turbine loads based on a stochastic description of the turbulent wind input.

To address this problem we reformulate an industry standard wind model into a reduced-order engineering model. The aim of our work is to develop a wind model that can generate a realistic wind field with appropriate (long-term) dynamic properties from considerably less random variables than the current models. In the last 3 decades numerous turbulent wind models have been proposed. Kleinhans et al. (2008) summarize a few. However, none of the previous models had an application in stochastic aerodynamic models in mind. Since random numbers can be generated very quickly, existing models rely on a large set of random variables to be used as a seed for a wind field realization. However, this random seed usually contains too many random variables to be applicable to a direct stochastic modelling of the aerodynamic wind turbine equations (path $\mathrm{C}$ in Fig. 1).

In the present study, we focus on a formulation for the IEC standard spectral wind description (IEC 61400-1, Ed. 3, 2005), so that it may be directly used for stochastic aerodynamic models. Veers' model (Veers, 1988) was chosen as a baseline and starting point. This model is widely used, for example in the stochastic wind simulator TurbSim (described by Jonkman and Kilcher, 2012), which synthesizes a sample of turbulent atmospheric wind from Veers' spectral formula- tion. Although it is well known that Veers' model does not capture all physical details of "real" atmospheric wind (e.g. Mücke et al., 2011; Morales et al., 2012; Lavely et al., 2012; Park et al., 2015), it is for many cases an appropriate engineering model (Nielsen et al., 2007). Due to its comparatively high independence of site-specific parameters, ease of use, and low resource requirements, Veers' model is the preferred model for many applications (Lavely et al., 2012). Moreover, it is endorsed by the governing wind turbine design standard (IEC 61400-1, Ed. 3, 2005) and thus is widely used in the wind energy industry. This underlines that its fidelity is accepted as a reasonable compromise in engineering practice for wind energy. As such, Veers' model provides a well-accepted foundation to base further development on. Note that our goal is not to improve on the known deficiencies of Veers' model but to arrive at a model that can generate a wind sample of comparable (and accepted) fidelity with significantly less random variables, geared towards eventual inclusion in a stochastic wind turbine simulation.

The following sections will first briefly review Veers' model to set the stage for the proposed modifications. Subsequently, the new reduced-order wind model is introduced, and finally results are presented, which confirm that key statistical properties (cross-correlation, covariance, auto- and cross-spectrum) are conserved by the new model. The paper concludes by giving direction for continued work on integrating the wind model into a turbine simulation and on refinements with other turbulent wind descriptions. To not overload this paper, the focus is solely on the details and validation of the stochastic wind inflow model itself. Interested readers should refer to Fluck and Crawford (2017a) for the basic stochastic aerodynamic model or Fluck and Crawford (2016b) for an example of how the reduced-order wind model is used to calculate stochastic loads on a stationary wing.

\section{Method}

Veers' method represents the established method for synthesizing turbulent wind (Nielsen et al., 2007; Lavely et al., 2012) and at the same time is the baseline for our contribution. Hence, the method is briefly summarized here to lay out the basics for the following work. For a complete introduction the reader is referred to Veers' original paper (Veers, 1988) and successive work, e.g. Kelley (1992), Nielsen et al. (2004), and Burton et al. (2011).

\subsection{Veers' method}

In Veers' spectral method, the wind speed time series $u_{k}(t)$ at each point $P_{k}, k=1 \ldots N_{P}$ in the sampled wind field is obtained through the inverse discrete Fourier transform of a set of discrete frequencies components from the double-sided 
(symmetric) spectrum $U_{m k}$ at $\omega_{m}=2 \pi f_{m}, m=-N_{F} \ldots N_{F}$ :

$u_{k}(t)=\sum_{m} U_{m k} e^{i \omega_{m} t}$

Here $m$ is used to index the frequency bins, and $k$ is used to index the points in space where wind speed data are recorded. Usually, the terms $U_{m k}$ are binned Fourier amplitudes centered at the frequency $\omega_{m}$, prescribed by the wind speed spectrum $S\left(\omega_{m}\right)$. In many cases, the Kaimal spectrum is used (Kaimal et al., 1972).

Following Veers' method (Veers, 1988), $U_{m k} \in \mathbb{C}$ contains not only the amplitude but also the random phase angles at $P_{k}$ for each frequency $\omega_{m}$. To obtain the desired coherence for all frequencies and between any two points in the wind field, all phase angles

$\theta_{m k}=\arctan \left(\frac{\operatorname{Im}\left(U_{m k}\right)}{\operatorname{Re}\left(U_{m k}\right)}\right)$

need to be correlated correctly. To achieve this, Veers starts with a set of $N_{R}=N_{F} \cdot N_{P}$ independent, uniformly distributed random variables $\xi_{j m} \sim U(0,1)$ and multiplies these with a weighting tensor $H_{j k m}$, obtained from the discrete cross-spectrum $S_{j k}\left(\omega_{m}\right)$ (given by the relevant design standard or physics model), to obtain the complex Fourier coefficients $U_{m k}$ for each frequency band $\omega_{m}$ :

$U_{m k}=\sum_{j=1}^{k} H_{j k m} e^{i 2 \pi \xi_{j m}}$.

Through Eq. (3) the phase angles at each point $P_{k}$ are related to the phases at all previously computed points $P_{j<k}$. Thus, correctly correlated Fourier coefficients are obtained, which can now be inserted into Eq. (1) to obtain a correlated wind field.

This method works well to generate multiple (deterministic) wind speed data sets at many points. However, as already noted by Veers in his original publication (Veers, 1988), Eq. (3) changes the amplitude of each Fourier coefficient, such that $\left|U_{m k}\right| \neq \sqrt{S_{k k}\left(\omega_{m}\right)}$ for all but the point computed first. Thus, the prescribed (e.g. Kaimal) spectrum $S_{k k}\left(\omega_{m}\right)$ is not conserved anymore at each point for any single realization; see Fig. 2. However, if spectra are averaged over either several points or several realizations, the wind field's average spectrum converges to the prescribed spectrum as $\lim _{N \rightarrow \infty} 1 / N \sum_{k=1}^{N}\left|U_{m k}\left(\omega_{m}\right)\right|=\sqrt{S_{k k}\left(\omega_{m}\right)}$, with $N$ the number of samples or realizations. This means the field is still stochastically homogeneous, as expected. However, for a stochastic analysis where only a limited number of samples might be used, this may pose a challenge. We introduce a reduced-order model based on phase angle increments. This model not only yields a significant reduction in random variables required to synthesize a stochastic wind field, but it also analytically preserves the prescribed spectrum at any single point for each realization (see Fig. 2 "phase increments").

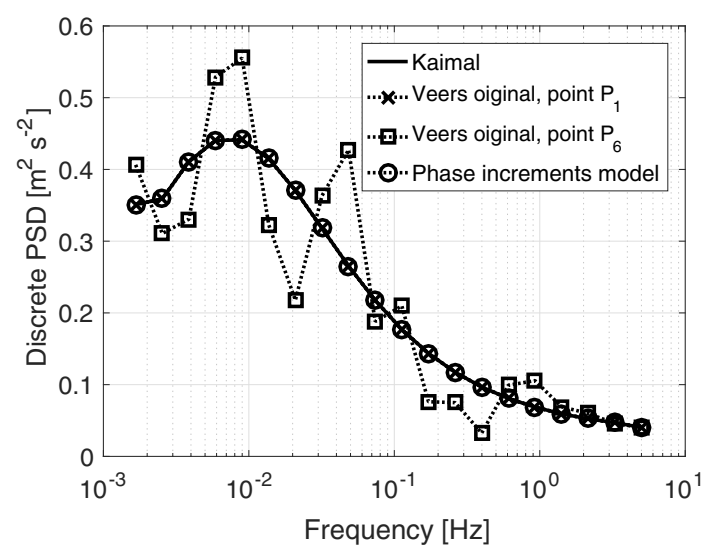

Figure 2. Raw wind spectra from a single wind speed sample; no averaging. Kaimal: the analytic spectrum; Veers: sample of the spectrum resulting from Eq. (3) at two different points $P_{1}$ and $P_{6}$; phase increments: the spectrum from the reduced-order phase increment model Eq. (8) (identical for all points). The values from Kaimal, Veers' original $\left(P_{1}\right)$, and the phase increment models all collapse to the same line.

\subsection{The reduced-order model with phase increments}

To arrive at a reduced-order model, we follow a two-step process. First is a reduction in the number of frequencies necessary for the spectral composition of the wind speed time series at a single point in space, which yields a reduction in the number of random phase angles associated with each frequency. This frequency reduction has been done before. For example, Fluck and Crawford (2017a) showed that with 10 frequencies from the IEC Kaimal spectrum, logarithmically spaced in $[0.003,5] \mathrm{Hz}$ (a $T=333 \mathrm{~s}$ sample, resolved at $10 \mathrm{~Hz}$, a reasonable time step for wind turbine simulations; cf. Bergami and Gaunaa, 2014), a realistic wind speed time series can be produced. The choice of 10 frequencies is not driven by physical arguments but by the fact that it is sufficient to obtain a wind speed time series similar to results from a full TurbSim simulation at $10 \mathrm{~Hz}$ for $10000 \mathrm{~s}$ (roughly $5 \times 10^{4}$ frequency bins), including a similar probability distribution (and thus turbulence intensity), as well as similar wind speed auto-correlation. On the other hand, 10 frequencies, and thus 10 random variables for the phase angles, is manageable as input to a stochastic model. However, when dealing with a wind field big enough to be used for wind turbine calculations, many points (for a $D=90 \mathrm{~m} \mathrm{di}$ ameter rotor, somewhere in the order of $15 \times 15$ points over the rotor disk are typically used) of correlated wind speed are necessary. The challenge is to extend this limited frequency wind description from a single point to a spatially varying wind field without excessively increasing the number of random variables required. Fung et al. (1992) introduced a wind model which describes both the spatial and the temporal dimension through Fourier modes. They reduced the number of modes down to as little as 38 ; however, the model 
then relied on several random numbers associated with each mode. Fung et al. (1992) did not report in detail how many random variables they used for their model, but the equations indicated that this number was still considerably larger than manageable by stochastic methods. The following paragraphs will introduce a new approach, which will allow us to create a stochastic wind field from a significantly reduced number of random variables, independently of the spatial size of the wind field, i.e. independently of the number of data points over the rotor disc.

In Veers' model the phase angle matrix $\boldsymbol{\Theta}=\left[\theta_{m k}\right]$ is populated with random numbers. We note that random phase angles in the rows and columns of $\boldsymbol{\Theta}$ carry out two distinctly different functions. At each individual point $P_{i}$ the different phase angles in the column vector $\left[\theta_{m}\right]_{i}=\boldsymbol{\Theta}_{i}$ generate constructive/destructive interference of the ensemble of base sinusoids. Thus, different realizations of $\boldsymbol{\Theta}_{i}$ generate the "gusty" nature of the wind speed time series at that point. This is indeed the streamwise and thus the temporal variability in the wind field. On the other hand, the wind speed structure in space, for example the fact that strong winds at one point correlate with strong winds at a nearby point, is captured through the relation of phase angles for the one particular frequency $\omega_{l}$ at different points $P_{i}$ and $P_{j}$ - that is in each row of $\boldsymbol{\Theta},\left[\theta_{k}\right]_{l}=\overline{\boldsymbol{\Theta}}_{l}$. This is the spatial variability in the wind.

While the phase angles at each point (the columns $\boldsymbol{\Theta}_{i}$ ) are uncorrelated, the phase angles between two points (the rows $\overline{\boldsymbol{\Theta}}_{l}$ ) have to be somehow dependent on each other to reproduce the spatial structure correctly. For two column vectors $\left[\theta_{m}\right]_{i}$ and $\left[\theta_{m}\right]_{j}$, this means while the entries within each column vector are uncorrelated $\left(\operatorname{cov}\left(\theta_{m k}, \theta_{n k}\right)=0\right)$, the elements within each vector are not independent, i.e. correlated $\left(\left|\operatorname{cov}\left(\theta_{m k}, \theta_{m l}\right)\right|>0\right)$; cf. Fig. 3. For wind, this correlation decreases with both increasing frequency and increasing distance. Based on these observation, we note the following for our use case of turbulent wind as input to dynamic wind turbine analysis.

1. The temporal variability (in the columns of $\boldsymbol{\Theta}$ ) is of primary importance, since it drives the dynamic excitation of the system under investigation. This is the structure of gusts and lulls, captured by the energy distribution in the frequency spectrum of the wind sample.

2. The spatial variability (in the rows of $\boldsymbol{\Theta}$ ) also needs to be represented correctly to yield representative wind loads, which eventually result in the correct integral loads. For example, at any instance when a sensor $A$ somewhere on the blade experiences an increased load, another sensor $B$ a certain distance away from $A$ needs to experience a load correctly correlated to the load at $A$. However, since there will necessarily be some averaging of the loads across the blades, this is of secondary importance.

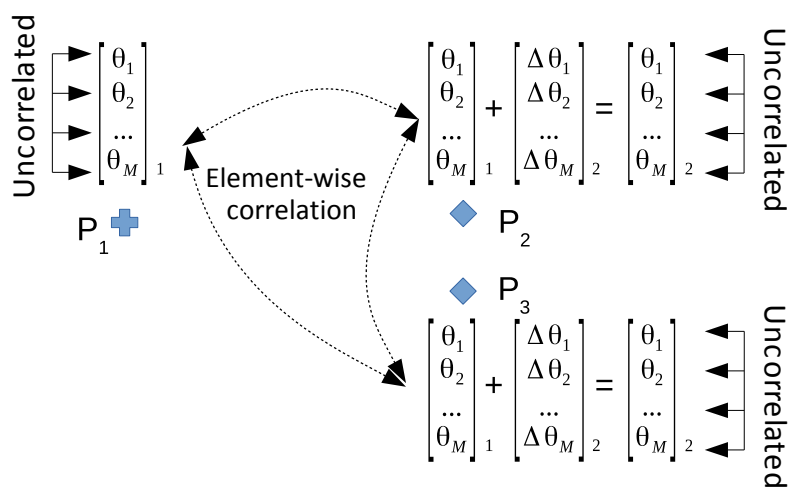

Figure 3. Schematic of random phase angle vectors and deterministic phase increments.

3. For each point, all elements in each column vector $\boldsymbol{\Theta}_{i}$ are independent (Fig. 3). However, the column vectors $\boldsymbol{\Theta}_{i}$ and $\boldsymbol{\Theta}_{j}$ at two points $P_{i}$ and $P_{j}$ are correlated in an element-wise way. This means the phases in each row vector $\overline{\boldsymbol{\Theta}}_{l}$ are not independent. Following Veers' method, only the elements in $\boldsymbol{\Theta}_{1}$ are independent, while the phases at all other points are mapped from independent and identically distributed random variables $\xi_{m i}$ such that they are correlated to the phases at the base point $P_{1}$ (and thus to each other); Eq. (3). This means there is more "randomness" in the columns of $\boldsymbol{\Theta}$ than in the rows - an important fact, which we will soon exploit.

To obtain a reduced-order model which requires fewer random variables, we propose splitting the complex Fourier coefficients $U_{m k}$ into a temporal and a spatial part. The temporal part will contain the amplitude of each Fourier mode as well as the random phase angles. It will therefore determine the structure of the wind speed sample in time. The spatial part will contain the phase correlation between different points across the wind field. It will thus set the wind field structure in space. To reflect this approach we can write

$U_{m k}=\underbrace{U_{m 1}}_{\text {temporal }} \cdot \underbrace{e^{i \Delta \theta_{m k}}}_{\text {spatial }}$.

The temporal part contains the amplitude according to the prescribed power spectrum $S\left(\omega_{m}\right)$ and a vector of random phase angles $\theta_{m 1}=2 \pi \xi_{m}$ at an arbitrary base point $P_{1}$ within the wind field:

$U_{m 1}=\sqrt{S\left(\omega_{m}\right)} e^{i \theta_{m 1}}$

with independent and identically distributed $\xi_{m} \sim U(0,1)$ as before. Similar to the wind speed increments used for wind interpolation by Fluck and Crawford (2016a), the spatial part is based on the idea of phase increments $\Delta \theta_{m k}$, which are specific to each point and each frequency relative to the base 
point $P_{1}$ :

$\Delta \theta_{m k}=\theta_{m k}-\theta_{m 1}$

The increment $\Delta \theta_{m k}$ holds the correlated phase information to generate the correct spatial structures. Since $\theta_{m k}$ and $\theta_{m 1}$ are random numbers, the increments $\Delta \theta_{m k}$ should be random, too. However, in contrast to Veers' approach of employing the cross-spectrum to map a set of uncorrelated random variables to a set of correlated phases for each point in the wind field, we neglect the random nature of $\Delta \theta_{m k}$ and consider the phase increments deterministic constants. This means that for different wind field realizations, the correlation coefficient between two points in space is fixed for each frequency (NB this is the stochastic correlation; it does not establish a deterministic one-to-one dependence of the wind speeds at two points). For example, for a frequency bin at $f_{1}=10 \mathrm{~Hz}$ and point $P_{4}, \Delta \theta_{14}$ has the same value for each realization. Similarly, for a frequency bin $f_{5}=0.1 \mathrm{~Hz}$ and the same point, $\Delta \theta_{54}$ once again always has the same value (but different from $\Delta \theta_{14}$ ).

Assuming $\Delta \theta_{m k}$ to be constant is the core assumption of the presented reduced-order model. It clearly is a simplification, but it is essential to arriving at a model reliant on a reduced number of random variables. The results presented in Sect. 3 will confirm the validity of this assumption. Note, moreover, that $\Delta \theta_{m k}$ only contains the spatial structure but not the temporal part. This means gusty features of the wind (lulls and gusts at different points) are still generated from random numbers; only the wind field's spatial correlation is fixed with each specific set of phase increments. Based on the three observations above (1-3), this seems justified for two reasons. Firstly, the phases in each row vector $\overline{\boldsymbol{\Theta}}_{l}$ are correlated, while the phases in each column vector $\boldsymbol{\Theta}_{i}$ are uncorrelated (3). This means there is more randomness in the temporal dimension then in the spatial dimension. Secondly, for the dynamic analysis of a wind energy device, the temporal part is of primary importance. While the spatial structures have to be represented correctly, their exact variability, however, can be considered secondary $(1,2)$.

It is important to note that focusing on the temporal part does not mean that each realization of the reduced-order wind field will exhibit the same spatial structure of gusts and lulls, i.e. that a gust at point $P_{i}$ would necessarily come with a lull at another point $P_{j}$. By contrast, the proposed method does not alter the original correlation between wind speeds at two points, which is generally smaller than unity. From a graphical point of view, gusts and lulls result from the interference of different frequency component sinusoids and phase offsets. Based on the specific realization $\theta_{m 1}$, the phase angles at each point $\theta_{m k}=\Delta \theta_{m k}+\theta_{m 1}$ will be different each time. Thus, the interference between the frequency components and consequently the structure of the gusts and lulls will be different with each different realization of phases at the base point $\theta_{m 1}$. Figure 6 , which will be discussed later, demonstrates this fact. Nonetheless, the proposed reduction of random variables necessarily causes a certain increase in dependence of wind speeds across the wind field. An investigation into how this dependence actually looks in detail will be the subject of future work.

Inserting Eqs. (5) and (6) into Eq. (4) yields the Fourier coefficients based on only one vector $\left[\theta_{m 1}\right]$ of random phases and the (auto-) spectrum:

$U_{m k}=\sqrt{S\left(\omega_{m}\right)} e^{i\left(\theta_{m 1}+\Delta \theta_{m k}\right)}$.

Substituting $\theta_{m 1}=2 \pi \xi_{m}$, Eq. (1) can be turned into our reduced-order model (with $\xi_{m} \sim U(0,1)$ as before):

$u_{k}(t)=\sum_{m} \sqrt{S\left(\omega_{m}\right)} e^{i\left(\omega_{m} t+2 \pi \xi_{m}+\Delta \theta_{m k}\right)}$.

Note that while Eq. (3) changes the amplitude of each Fourier coefficient and thus distorts the spectrum at each point, Eq. (8) fully conserves the spectrum.

In contrast to Veers' original model, where $N_{R}=N_{F} \cdot N_{P}$, in the reformulated model $N_{R}=N_{F}$. This means the number of random variables $N_{R}$ only depends on the number of frequencies $N_{F}$ used for the wind speed Fourier series, not on the number of wind speed measurement points $N_{P}$ in the 3-D wind field. With the available strategies to reduce the number of frequencies required in a spectral wind model Eq. (8) now allows the expression of a turbulent wind field consistent with Veers' model but with significantly fewer frequencies.

What remains is to obtain the phase angle increments $\Delta \theta_{m k}$. Since these determine the cross-correlation between any two points in the wind field, and since the crosscorrelation and the cross-spectrum are linked as a Fourier transform pair (correlation theorem; see, e.g., Kauppinen and Partanen, 2011), it should be possible to analytically generate one set (one realization) of phase increments directly from the cross-spectrum. For now, however, we extract one phase angle increment set from one realization of Veers' Eq. (3), with an analytic solution left to future work.

\section{Results and discussion}

In the following, we will take a closer look at statistical metrics of the synthetic reduced-order wind field. As mentioned earlier our goal is not to develop a more physically faithful wind model but rather to reduce the number of random variables required while retaining a similar fidelity to the methods currently in use. TurbSim (Jonkman and Kilcher, 2012) is widely used in industry and is the de facto standard to generate synthetic wind fields for wind turbine analysis. Hence, we use TurbSim wind speed data sets as the benchmark. In the following, we compare results obtained from TurbSim to two different reduced-order models.

The first is our implementation of Veers' model, which allowed us to freely choose the number of frequencies at each data point and the frequency binning. Following Veers this implementation relies on an inverse discrete Fourier transform with random phase angles at each frequency bin. This 
Table 1. Comparison of random numbers used in different wind models for a common grid size.

\begin{tabular}{lrrr}
\hline & TurbSim & Veers $_{\text {red }}$ & Veers \\
& $10 \mathrm{~min}$ & $10 \mathrm{~min}$ & $10 \mathrm{~min}$ \\
Sample length & $15 \times 15$ & $15 \times 15$ & $15 \times 15$ \\
Grid size $N_{P y} \times N_{P z}$ & $\sim 3000$ & 20 & 20 \\
Frequencies $N_{F}$ & & & \\
Total number of random & $6.75 \times 10^{5}$ & 4500 & 20 \\
Variables $N_{R}$ & & & \\
\hline
\end{tabular}

model was validated directly against TurbSim. If many frequencies are used and identical phase angles are enforced perfect agreement of the resulting data set was found as expected. As shown by Fluck and Crawford (2017a), the wind speed time series at a single point for a $333 \mathrm{~s}$ sample can be well represented with $N_{f}=10$ logarithmically spaced frequencies, which allowed a more efficient representation of the wind and its spectrum. We set $f=\omega /(2 \pi)=$ $\left[f_{m}\right] \in\left[f_{1}, f_{N_{F}}\right]=[0.003,5] \mathrm{Hz}$ with $f_{m}=10^{a_{m}}$ and $a_{m}=$ $\log _{10}\left(\frac{f_{N_{F}}}{f_{1}}\right) \frac{m-1}{N_{F}-1}$ for $m=1, \ldots, N_{F}$. For better comparison (equal sample length $T=600 \mathrm{~s}$ ), we use $N_{F}=20$ frequencies in $\boldsymbol{f}=\left[f_{m}\right] \in[1 / 600,5] \mathrm{Hz}$. The results of this model are labelled "Veers red" in the following discussion. This model does not include new theory, yet it is a critical step between TurbSim (and thus Veers' original model) and our reduced-order model. The second model presented is our reduced-order model as described above (Eq. 8). The newly introduced theory of deterministic phase increments $\Delta \theta$ is employed here, together with a limited number of $N_{F}=20$ frequencies and thus a reduced number of stochastic variables $N_{R}=20$. These results are labelled "Veers ${ }_{\text {red, }} \Delta \theta$ ".

Table 1 gives a comparison of the three models. Note particularly the total number of random variables required by each model, assuming a typical grid resolution in the order of $15 \times 15$ points over a rotor disk of $D=90 \mathrm{~m}$ diameter. While the use of a limited set of frequencies (Veers red$_{\text {) }}$ yields a noticeable reduction in random numbers, for a turbulent wind field with several wind speed data points in $x$ and $y$ directions, this alone is not enough to arrive at a wind model with few enough random numbers to be applicable in a stochastic method (several dozen random variables to be tractable). Only the additional introduction of deterministic phase increments (Veers $s_{\text {red, }} \Delta \theta$ ) to decouple the number of random variables from the number of wind speed data points reduces the number of random variables drastically enough to obtain a wind model which can be reasonably handled by a stochastic method.

Since the goal here is not to calculate wind turbine loads, but to merely asses the quality of the reduced-order wind model, we used a dummy wind field generated on $N_{P y} \times$ $N_{P z}=5 \times 3=15$ points located on a regular grid as depicted in Fig. 4. This is fewer points than the usual grid for the analysis of a modern $D=90 \mathrm{~m}$ rotor diameter wind turbine.

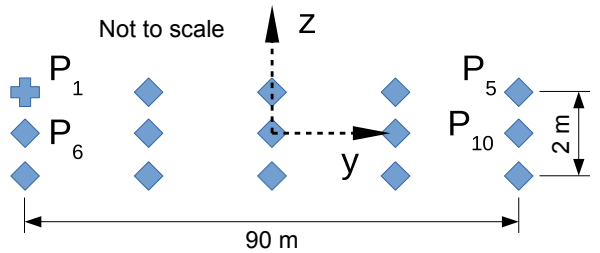

Figure 4. Schematic of grid points of wind speed data (minimal test case). We arbitrarily chose the right-hand top point $\left(P_{1}\right)$ to be the base point.

However, the reduced number of grid points enabled us to solve the equations quickly with all models and more clearly illustrate the method. At the same time, the configuration of Fig. 4 still allowed us to study both the wind speed time series of points in close proximity (e.g. $P_{1}$ and $P_{6}$ ), as well as at more distant points (e.g. $P_{1}$ and $P_{5}$ ). The origin of the wind field was located $100 \mathrm{~m}$ above ground with mean wind speed $\bar{u}=10 \mathrm{~m} \mathrm{~s}^{-1}$ and no wind shear. The IEC class A normal turbulence model with a Kaimal spectrum and homogeneous turbulence was used (IEC 61400-1, Ed. 3, 2005). Data were sampled at $10 \mathrm{~Hz}$. We arbitrarily chose the top left-hand point $\left(P_{1}\right)$ as the base point.

Figure 5 shows realizations of the wind speed time series sampled at four points $\left(P_{1}, P_{5}, P_{6}\right.$, and $P_{10}$ in Fig. 4) from the three different models. For each model the samples are generated from different random seeds. Thus, the time series are not identical. Still, it can be seen that the fundamental structures are conserved through both reduced-order models. In particular, even if wind samples are synthesized with only 20 random numbers and deterministic phase increments (Veers ${ }_{\text {red, }} \Delta \theta$ ), the wind speeds at two points in close proximity $\left(P_{1}\right.$ and $P_{6}$, or $P_{5}$ and $\left.P_{10}\right)$ are highly correlated, while at more distant points (e.g. $P_{1}$ and $P_{5}$ ) the correlation is weaker. It is important to note that this holds not only for points in relation to the base point but for all point pairs. For example, points $P_{5}$ and $P_{10}$ are both far away from the base point, but close to each other. As expected, the wind speeds at these two points are well correlated.

Figure 6 shows three realizations of wind speed time series plots at three points obtained from the new phase increment model (Veers red, $\Delta \theta$ ), Eq. (8). The phase increments are considered deterministic, and $\Delta \theta_{m k}$ is fixed for all realizations. The randomness enters the time series only via random phase angles at the base point $P_{1}$ with $\theta_{m 1}=2 \pi \xi_{m}$. As can be seen from the figure, this does not result in a complete determination of the spatial relation between wind speeds at different points, since the samples still contain different gusts and lulls at different instances in time.

Beyond this qualitative visual comparison of the wind speed time series, the remainder of this section will show that the phase increment model produces the same statistics as Veers' original model (with only 20 frequencies) as well 

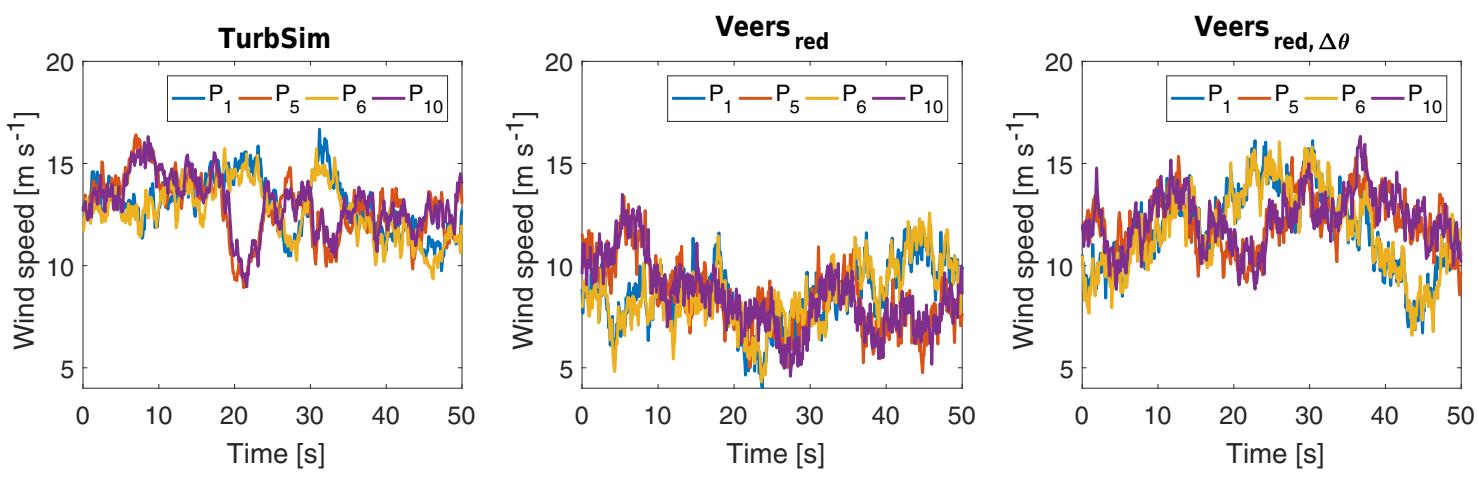

Figure 5. Three $50 \mathrm{~s}$ excerpts of a wind speed time series samples at four points generated from different models and different random seeds. TurbSim: NREL's original TurbSim model; Veers ${ }_{\text {red }}$ : Veers' model with a limited number of frequencies $\left(N_{F}=20\right)$; Veers ${ }_{\text {red, }} \Delta \theta$ : Veers' model with a limited number of frequencies and deterministic phase increments. See Table 1 for additional information.
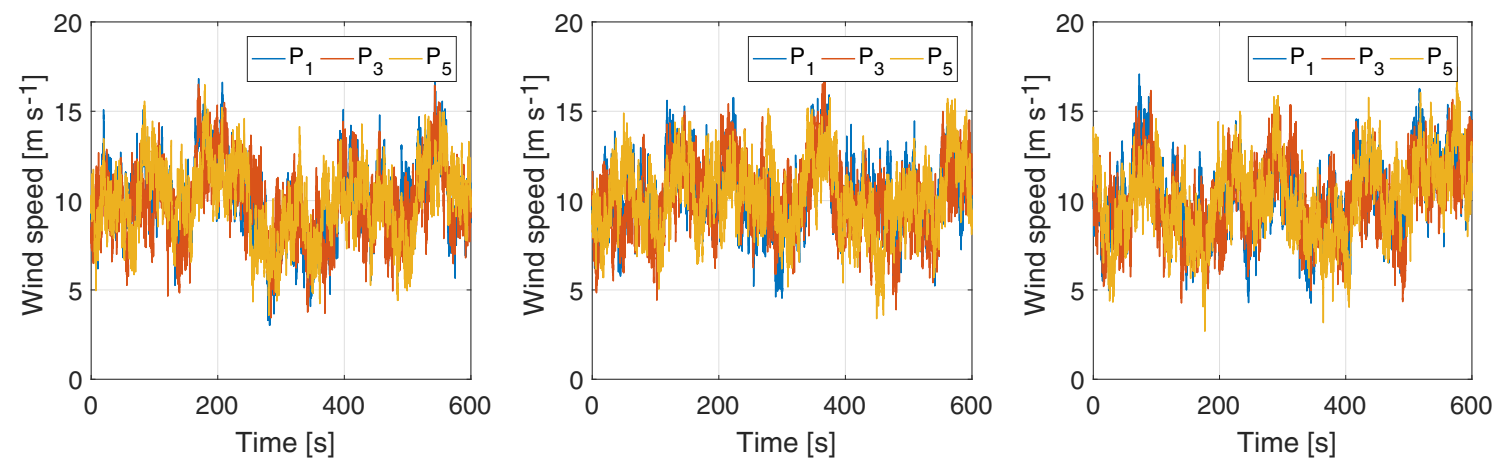

Figure 6. Three realizations of wind speed time series at three points generated from the new reduced-order model with fixed phase increments (Veers $\mathrm{red}, \Delta \theta$ ).

as the full TurbSim model (with the full set of frequencies) for the most important metrics.

\subsection{Cross-correlation}

Figure 7 compares the cross-correlation for two different point pairs $-P_{1}-P_{5}(90 \mathrm{~m}$ apart $)$ and $P_{1}-P_{6}(1 \mathrm{~m}$ apart $)-$ as obtained from six $99 \mathrm{~s}$ windows from a $600 \mathrm{~s}$ sample from our reduced model with fixed phase increments (Veers ${ }_{\text {red, }} \Delta \theta$ ), from Veers' model with 20 frequencies (Veers red $_{\text {), and from }}$ the full TurbSim simulation. To reduce noise and compare meaningful (rather than possibly extreme) values, the results are presented as averages of 100 realizations from different random seeds for both phase angles and phase increments.

As can be seen from Fig. 7, the cross-correlation in general agrees very well for both the close points and the distant point pair. The results from our implementation of Veers' model and from the phase increment model are almost identical and hence difficult to distinguish. Note that the TurbSim data are smoother, presumably due to the significantly higher number of frequencies contained in the TurbSim data set.

Further investigation with the pair $P_{5}$ and $P_{10}$, two points close to each other but far away from the base point $P_{1}$

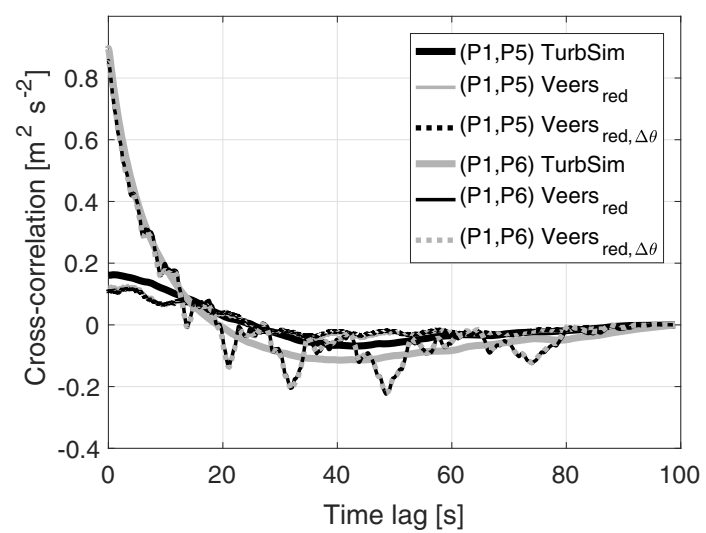

Figure 7. Wind speed cross-correlation for two point pairs generated from different models: a close pair $\left(P_{1}, P_{6}\right)$ and a distant one $\left(P_{1}, P_{5}\right)$.

(not included in Fig. 7), shows that for all three models the cross-correlation is almost identical to the curve for $P_{1}-P_{6}$. This confirms that with our phase increment model the crosscorrelation of the homogeneous turbulence field, and with it the length scale of spatial structures, is indeed only depen- 
dent on the distance between two points but not on the two specific points themselves.

\subsection{Covariance}

Now we look at the covariance as a function of the distance between two points and compare data from TurbSim to Veers' model (Veers ${ }_{\text {red }}$ ) and to our reduced model with phase increments (Veers $r$ red, $\Delta \theta$ ). As above we use averages from $99 \mathrm{~s}$ windows out of 100 realizations of $600 \mathrm{~s}$ samples.

From Fig. 8 it can be seen that the covariance from all three model agrees fairly well. Our implementations of Veers' model, Veers red $_{\text {and Veers }}$ red, $\Delta \theta$, which both use a limited set of frequencies, agree almost perfectly. The TurbSim version with the full set of roughly 3000 frequencies, on the other hand, yields slightly different covariance. A more detailed investigation reveals the reason for this: the covariance depends on the cross-spectrum and thus the spectrum at each individual point. Consequently, the discrepancy between the covariance functions is connected to the fact that Veers' model distorts the spectrum at each individual point, such that with Eq. (3) $\left|U_{m k}\right|=\sqrt{\widetilde{S}_{m k}} \neq \sqrt{S_{m k}}$ (see discussion in Sect. 2.1). When we replace $S$ in our implementation by the distorted spectrum $\widetilde{S}$ at each particular point $P_{k}$ in Eq. (8), all three curves do match. However, $\widetilde{S}$ does not in fact represent the prescribed Kaimal spectrum. Thus, we conclude that our phase increment model actually represents the desired covariance better than Veers' original model and TurbSim.

\subsection{Power spectra}

Wind speed power spectra are again obtained as average from 100 realizations (from 100 different random seeds). However, this time $6000 \mathrm{~s}$ were sampled to obtain sufficiently long data sets for a proper resolution of the lowfrequency components. Note that the same set of 20 frequencies $\left[f_{k}\right] \in[1 / 600,5] \mathrm{Hz}$ are used for both the 20-frequency (Veers $\left.\mathrm{red}_{\mathrm{r}}\right)$ and the phase increment $\left(\right.$ Veers $\left._{\mathrm{red}}, \Delta \theta\right)$ implementations. Hence, the $T=6000 \mathrm{~s}$ signal repeats after $600 \mathrm{~s}$. The spectrum is binned into discrete bins of frequencies $f_{m}$ equal to the logarithmically spaced frequencies initially used to generate the wind speed time series.

The wind speed auto-spectrum is included in Fig. 2. By definition (Eq. 8), the reduced-order model produces the prescribed (auto-) spectrum exactly. Figure 9 shows a comparison of the cross-spectra estimates for different point pairs obtained through Welch's periodogram method (Welch, 1967) employed on the full $6000 \mathrm{~s}$ samples with no extra windowing. We study the base point and its closest neighbor $\left(P_{1}-P_{6}\right)$, the base point and a point far away $\left(P_{1}-P_{5}\right)$, and a point pair close together but far away from base point $\left(P_{5}-P_{10}\right)$. For reference, the prescribed Kaimal spectrum $S$ is included, as well as the analytic cross-spectrum obtained by

$S_{i j}=\operatorname{coh} \sqrt{S_{i i} S_{j j}}$,

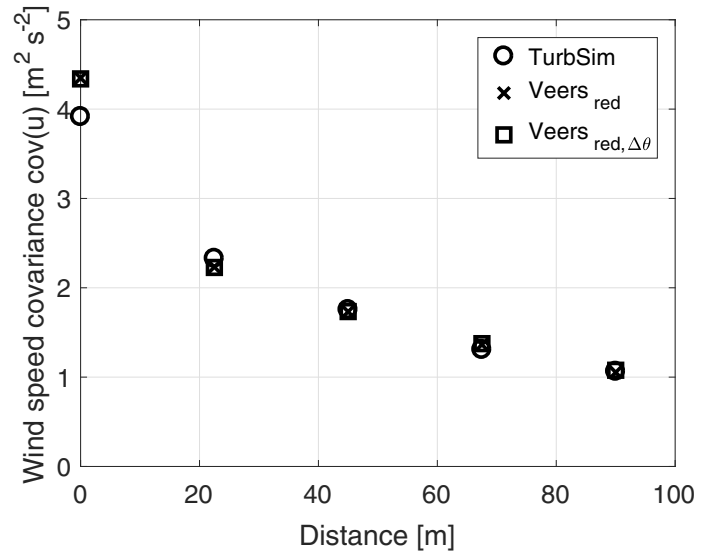

Figure 8. Wind speed covariance for points different distances apart.

with the (auto-) spectra $S_{i i}=S$ and the coherence function coh as defined by the standard IEC 61400-1, Ed. 3 (2005).

Again, the phase increment model (Veers $s_{\text {red, }} \Delta \theta$ ) in all cases reproduces the analytic spectrum well with only 20 random variables. This time, however, the TurbSim results do not match as well. The reason is that TurbSim chooses the lowest frequency $f_{1}$ and the frequency bin width $\Delta f$ such that $\Delta f=f_{1}=1 / T$ and thus uses a wider frequency band for the first bin compared to our logarithmically spaced bins. When re-binning to the logarithmic range this results in excess power (and an artificial peak) in the first bin and hence less power in higher-frequency bins. Note, however, that this is an artifact of the discrete spectrum and the frequency binning and not a discrepancy in the underlying data.

\subsection{Outlook: wind turbine rotor blade loads}

To further assess the validity of the reduced-order wind model, loads were calculated for one single blade on a threebladed $R=35 \mathrm{~m}$ diameter wind turbine rotor spinning at a tip speed ratio $\lambda=6.1$. Loads were obtained at $\Delta t=0.1 \mathrm{~s}$ time steps through a simple blade element momentum model supplied with wind generated either from TurbSim or from our reduced model with fixed phase increments (Veers red, $\Delta \theta$ ) on a $15 \times 15$ grid of data points over the rotor disc. The hub height is set to $h_{\text {hub }}=90 \mathrm{~m}$, with the hub height mean wind speed $\bar{u}=12 \mathrm{~m} \mathrm{~s}^{-1}$, the power law wind shear with power law exponent $a=0.2$ (according to Jonkman and Kilcher, 2012), and the IEC normal turbulence model, class A (IEC 61400-1, Ed. 3, 2005). Figure 10 shows the probability distribution $p(T)$ of thrust loads $T$ on one blade calculated from 100 realizations of a $600 \mathrm{~s}$ wind field. TurbSim used the full set of roughly 3000 frequencies at each of the $15 \times 15$ grid points. The reduced-order model, on the other hand, relied on only 20 frequencies with all 100 realizations generated from one set of fixed phase increments. It can be seen, that the reduced-order model, although relying on significantly 

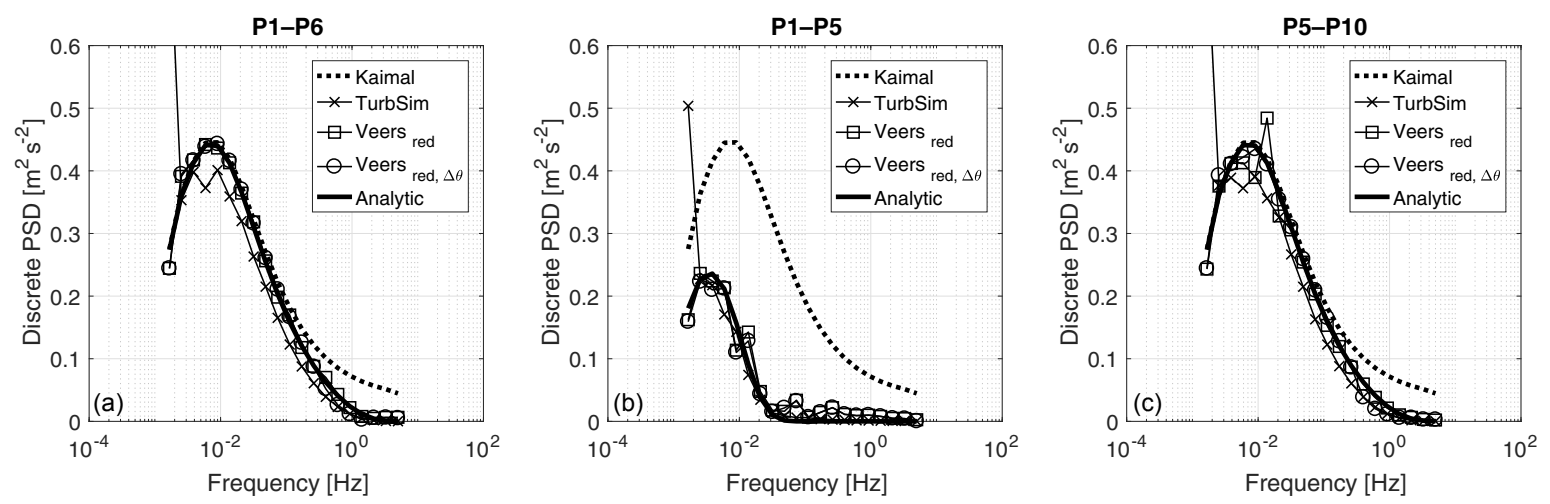

Figure 9. Wind speed cross power spectral density for three point pairs from different models, together with the analytic results (Eq. 9) and the prescribed Kaimal auto-spectrum. (a) The base point and its closest neighbor. (b) The base point and a point far away. (c) A point pair close together but far away from base point.

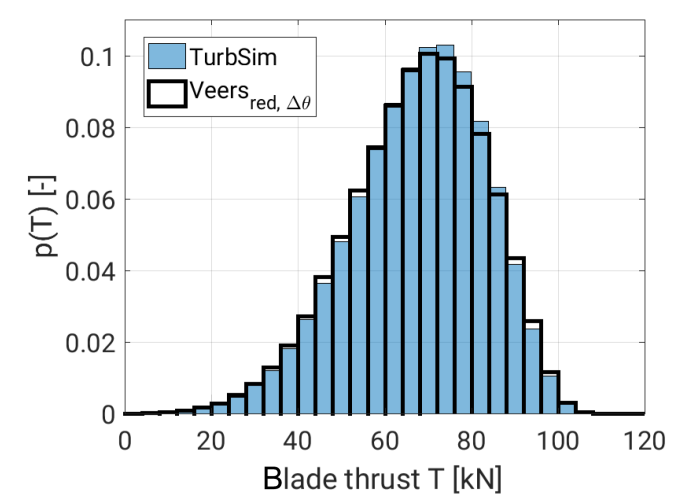

Figure 10. Blade thrust load probability distribution from a blade element momentum (BEM) model based on wind fields generated with either TurbSim or from the reduced-order Veers' model with constant phase increments.

fewer random variables $\left(N_{R}=6.75 \times 10^{5}\right.$ versus $N_{R}=20$ for each realization; see Table 1), produces almost the same load probability distribution. In a forthcoming publication (Fluck and Crawford, 2017b), we will use the reduced-order wind model presented here to develop a stochastic wind turbine blade load model (BEM). This publication will also discuss these blade load PDFs in greater detail.

\subsection{Discussion}

As shown in this section the phase increments wind model presented in Sect. 2.2 can reproduce important statistics (both of a wind field, as well as for resulting wind loads) with the accuracy comparable to the full model. At the same time, the phase increments model requires significantly less random variables. As indicated by Fig. 6 the phase increments model does not produce identical spatial structures with each realization, even thought a large part of the spatial randomness is neglected in Eq. (8). This further illustrates the method's ability to retain important stochastic information. Like Veers' model, our method relies on a spectral representation of a random process. The wind field is generated through an inverse Fourier transform with random phase increments. Thus, the underlying process is (per definition) assumed to be stationary. As with any spectral model, singular transient (and as such deterministic) events like fronts or downbursts can consequently not be modelled directly. It might be possible to model these events through the superposition of a discrete deterministic event with our random model. This could be similar to Chay et al. (2006), who superposes a deterministic downburst profile over an ARMA model for the turbulent fluctuations. However, it is our goal to derive a formulation for wind as input to stochastic models. These models will have difficulties dealing with singular deterministic events themselves. Hence, we doubt if progressing along this way would be beneficial; nonetheless, this could obviously be the subject of a future study.

The results from Sect. 3.1-3.3 are generated from a set of 100 different phase increments generated from 100 different random seeds. This was necessary because, due to the random equations, it was not possible to compare the results from a single realization. This might have resulted in uncharacteristically bad (or good) agreement only by the chance of comparing "bad" (or "good") realizations. Instead, only the averages over multiple realizations could be compared. For a stochastic analysis as outlined in the introduction, however, only a very limited set of phase increment realizations would be used. Hence, some part of the randomness of the wind field will be lost. This is the price to be paid for using a reduced-order model. In Sect. 2.2 we justify this choice. The results, particularly Figs. 6 and 10, support the notion that a very limited set of phase increment realizations, or even a single one, can be sufficient. It is still to be determined, however, how many sets will actually be necessary for adequate results and how the associated reduction in randomness influences the relevant output quantities, e.g. the resulting loads 
for a wind turbine analysis, especially the probability of extreme loads. Preliminary results for wind turbine blade loads calculated from a blade element momentum model indicate that only one single set of phase increments is sufficient to obtain almost the same statistical load distribution as from the conventional analysis based on standard TurbSim wind fields.

\section{Conclusions}

Stochastic analysis and uncertainty quantification are very active fields of research in engineering with the developed methods increasingly adopted by industry. To enable practitioners to apply these methods to wind turbine aerodynamics and more generally wind loading analysis on various structures, we presented a new method, which significantly reduces the number of random variables used in the wind model. This reduction is critical because the computational effort of the common stochastic solutions is very sensitive to the number of random variables involved.

The model introduced here employs a separation of the temporal (correlation in time) and spatial (coherence in space) part of the random dimension of turbulent wind. While the temporal part is still determined from random vari- ables, the spatial part is collapsed into deterministic phase increments. Thus, the number of random variables is reduced by several orders of magnitude compared to the commonly used model developed by Veers and implemented in TurbSim, currently the (de facto) standard tool for synthetic wind generation. A comparison of the most important stochastic metrics (cross-correlation, covariance, auto- and cross-spectrum) showed that the reduced-order model based on phase increments still reproduces these metrics as accurately as Veers' equations or TurbSim. Moreover, preliminary results were presented, which indicate that the reducedorder wind model based on phase increments also preserves wind turbine blade loads well. A detailed study quantifying the impact of using deterministic phase increments on the overall statistics of wind turbine loads is yet to be carried out. Subsequent to the implementation of this reduced-order wind model in a full wind turbine simulator, which is the focus of ongoing work, these ultimate questions can be addressed.

Data availability. Underlying data are obtained from TurbSim and custom implementations of the spectral wind models. TurbSim input files as well as copies of the implementation of the spectral models may be obtained from the authors upon request. 
Appendix A: Nomenclature

\begin{tabular}{ll}
\hline Latin letters: & \\
\hline$e$ & Euler's number \\
$f=\left[f_{m}\right]$ & frequency \\
$i=\sqrt{-1}$ & imaginary unit \\
$N_{F}$ & number of frequencies \\
$N_{P}$ & number of wind speed points \\
$N_{R}$ & number of random variables \\
$P$ & a point in Euclidean space \\
$S_{k k}$ & (auto) power spectrum \\
$S_{k j}$ & cross power spectrum \\
$t$ & time \\
$U$ & wind speed Fourier coefficient \\
$u$ & wind speed \\
\hline Greek letters: & \\
\hline$\theta$ & phase angle \\
$\Delta \theta$ & phase angle increment \\
$\xi$ & random number \\
$\omega=\left[\omega_{m}\right]$ & angular frequency \\
\hline Indices: & \\
\hline$j, k$ & points in space \\
$m$ & frequencies \\
\hline
\end{tabular}


Competing interests. The authors declare that they have no conflict of interest.

Acknowledgements. We gratefully acknowledge the funding provided for this study by the Pacific Institute for Climate Solutions (PICS), the German Academic Exchange Service (DAAD), and the Natural Sciences and Engineering Research Council of Canada (NSERC).

Edited by: Horia Hangan

Reviewed by: two anonymous referees

\section{References}

Bergami, L. and Gaunaa, M.: Analysis of aeroelastic loads and their contributions to fatigue damage, J. Phys. Conf. Ser., 555, 67-76, https://doi.org/10.1088/1742-6596/555/1/012007, 2014.

Bertagnolio, F., Rasmussen, F., Sørensen, N. N., Johansen, J., and Madsen, H. A.: A stochastic model for the simulation of wind turbine blades in static stall, Wind Energy, 13, 323-338, https://doi.org/10.1002/we.342, 2010.

Burton, T., Jenkins, N., Sharpe, D., and Bossanyi, E.: Wind energy handbook, Wiley, Chichester, West Sussex, UK, 2011.

Chay, M., Albermani, F., and Wilson, R.: Numerical and analytical simulation of downburst wind loads, Eng. Struct., 28, 240-254, 2006.

Desai, A. and Sarkar, S.: Analysis of a nonlinear aeroelastic system with parametric uncertainties using polynomial chaos expansion, Math. Probl. Eng., 2010, 379472, https://doi.org/10.1155/2010/379472, 2010.

Doubrawa, P., Barthelmie, R. J., Wang, H., and Churchfield, M. J.: A stochastic wind turbine wake model based on new metrics for wake characterization, Wind Energy, 20, 449-463, https://doi.org/10.1002/we.2015, 2017.

Fluck, M. and Crawford, C.: Minimizing errors in interpolated discrete stochastic wind fields, J. Wind Eng. Ind. Aerod., 152, 1522, https://doi.org/10.1016/j.jweia.2016.02.007, 2016a.

Fluck, M. and Crawford, C.: A stochastic aerodynamic model for stationary blades in unsteady 3-D wind fields, J. Phys. Conf. Ser., 753, 2410-2420, https://doi.org/10.1088/17426596/753/8/082009, 2016b.

Fluck, M. and Crawford, C.: Fast analysis of unsteady wing aerodynamics via stochastic models, AIAA J., 55, 719-728, https://doi.org/10.2514/1.J054983, 2017a.

Fluck, M. and Crawford, C.: A fast stochastic solution method for the Blade Element Momentum equations for long-term load assessment, Wind Energy, https://doi.org/10.1002/we.2148, online first, 2017b.

Fung, J. C. H., Hunt, J. C., Malik, N., and Perkins, R.: Kinematic simulation of homogeneous turbulence by unsteady random Fourier modes, J. Fluid Mech., 236, 281-318, 1992.

Guo, Q.: Incorporating stochastic analysis in wind turbine design: data-driven random temporal-spatial parameterization and uncertainty quantication, $\mathrm{PhD}$ thesis, Iowa State University, Ames, Iowa, USA, 2013.
IEC 61400-1, Ed. 3: International Standard 61400-1 (3rd edition): Wind turbines, Part 1: Design requirements, International Electrotechnical Commission, Geneva, Switzerland, 2005.

Jonkman, J. and Kilcher, L.: TurbSim user's guide: Version 1.06.00, Technical Report Draft Version, National Renewable Energy Laboratory, Golden, Colorado, USA, 2012.

Kaimal, J., Wyngaard, J., Izumi, Y., and Coté, O.: Spectral characteristics of surface-layer turbulence, Q. J. Roy. Meteor. Soc., 98, 563-589, 1972.

Kauppinen, J. and Partanen, J.: Fourier transforms in spectroscopy, Wiley-VCH, Berlin, Germany, 2011.

Kelley, N. D.: Full vector (3-D) inflow simulation in natural and wind farm environments using an expanded version of the SNLWIND (Veers) turbulence code, Tech. Rep. NRELtrP-4425225, National Renewable Energy Laboratory, Golden, Colorado, USA, 1992.

Kleinhans, D., Friedrich, R., Schaffarczyk, A. P., and Peinke, J.: Synthetic turbulence models for wind turbine applications, in: Progress in Turbulence III: Proceedings of the iTi Conference in Turbulence 2008, edited by: Peinke, J., Oberlack, M., and Talamelli, A., Springer, Berlin, Heidelberg, 111-114, https://doi.org/10.1007/978-3-642-02225-8_26, 2008.

Lavely, A., Vijayakumar, G., Brasseur, J., Patterson, E., and Kinzel, M.: Comparing unsteady loadings on wind turbines using TurbSim and LES flow fields, in: 50th AIAA Aerospace Sciences Meeting and Exhibit, AIAA, 9-12 January 2012, Nashville, Tennessee, USA, https://doi.org/10.2514/6.2012-818, 2012.

Le Maître, O. P. and Knio, O. M.: Spectral methods for uncertainty quantification: with applications to computational fluid dynamics, Springer, https://doi.org/10.1007/978-90-481-3520-2, 2010.

Majda, A. J. and Branicki, M.: Lessons in uncertainty quantification for turbulent dynamical systems, Discrete Cont. Dyn. S., 32, 3133-3221, https://doi.org/10.3934/dcds.2012.32.3133, 2012.

Morales, A., Wächter, M., and Peinke, J.: Characterization of wind turbulence by higher-order statistics, Wind Energy, 15, 391-406, https://doi.org/10.1002/we.478, 2012.

Moriarty, P.: Database for validation of design load extrapolation techniques, Wind Energy, 11, 559-576, https://doi.org/10.1002/we.305, 2008.

Mücke, T., Kleinhans, D., and Peinke, J.: Atmospheric turbulence and its influence on the alternating loads on wind turbines, Wind Energy, 14, 301-316, https://doi.org/10.1002/we.422, 2011.

Najm, H. N.: Uncertainty quantification and polynomial chaos techniques in computational fluid dynamics, Annu. Rev. Fluid Mech., 41, 35-52, https://doi.org/10.1146/annurev.fluid.010908.165248, 2009.

Nielsen, M., Larsen, G. C., Mann, J., Ott, S., Hansen, K. S., and Pedersen, B. J.: Wind simulation for extreme and fatigue loads, Technical Report Risö-R-1437 (EN), Ris $\varnothing$, Roskilde, Denmark, 2004.

Nielsen, M., Larsen, G. C., and Hansen, K. S.: Simulation of inhomogeneous, non-stationary and non-Gaussian turbulent winds, J. Phys. Conf. Ser., 75, 602-610, https://doi.org/10.1088/17426596/75/1/012060, 2007.

Padrón, A. S., Stanley, A. P. J., Thomas, J. J., Alonso, J. J., and Ning, A.: Polynomial chaos for the computation of annual energy production in wind farm layout optimization, J. Phys. Conf. Ser., 753, 032021, https://doi.org/10.1088/1742-6596/753/3/032021, 2016. 
Park, J., Manuel, L., and Basu, S.: Toward isolation of salient features in stable boundary layer wind fields that influence loads on wind turbines, Energies, 8, 2977-3012, https://doi.org/10.3390/en8042977, 2015.

Sudret, B.: Uncertainty propagation and sensitivity analysis in mechanical models - Contributions to structural reliability and stochastic spectral methods, Habilitation a diriger des recherches, Université Blaise Pascal, Clermont-Ferrand, France, 2007.

Sullivan, T. J.: Introduction to uncertainty quantification, Springer International Publishing Switzerland, Cham, Heidelberg, New York, Dordrecht, London, 2015.

Tibaldi, C., Henriksen, L. C., and Bak, C.: Investigation of the dependency of wind turbine loads on the simulation time, in: Proceedings of EWEA 2014, European Wind Energy Association (EWEA), Barcelona, Spain, 2014.
Veers, P. S.: Three-dimensional wind simulation, Technical Report SAND88-0152, UC-261, Sandia National Labs, Albuquerque, New Mexico and Livermore, California, USA, 1988.

Welch, P.: The use of fast Fourier transform for the estimation of power spectra: A method based on time averaging over short, modified periodograms, IEEE T. Acoust. Speech, 15, 70-73, https://doi.org/10.1109/TAU.1967.1161901, 1967.

Zwick, D. and Muskulus, M.: The simulation error caused by input loading variability in offshore wind turbine structural analysis, Wind Energy, 18, 1421-1432, https://doi.org/10.1002/we.1767, 2015 . 